\title{
JS02-01
}

\section{ANTIPSYCHOTICS IN ANXIETY DISORDERS}

\section{Allgulander}

Clinical Neuroscience, Karolinska Institutet, Huddinge, Stockholm, Sweden

In 1970 antipsychotics (haloperidol, levomepromazine, thiothixene) were recommended in Sweden as 1 st line treatment of anxiety in organic brain syndromes, psychoasthenia, and substance abuse. The benzodiazepines came to dominate treatment of anxious patients in the 1970s and are still appreciated for their rapid anxiolytic effect, low clinical toxicity and low price. Evidence-based guidelines, however, recommend the SSRIs, venlafaxine, and pregabalin as first line treatment in anxiety disorders.

Studies are currently ongoing to assess the utility of new antipsychotics in primary anxiety disorders: quetiapine, risperidone, aripiprazole, ziprazidone, and olanzapine. This is justified in the light of that a large proportion of these patients, about $1 / 3$, do not respond to $1^{\text {st }}$ line treatments or discontinue due to adverse drug effects. Augmentation therapy with antipsychotics may enhance response rates. Primary anxiety disorders may include psychotic symptoms such as dissociation, or severe insomnia, that may be targeted with these medications.

Another important patient category are patients with substance use disorders, particularly alcohol, in whom there are few or no controlled studies of conventional anxiolytics. Anxiety is also common among patients with psychoses, bipolar disorder, ADHD or borderline personality disorder, and few controlled studies have addressed that issue.

Emerging reports indicate that new antipsychotics may be useful as $1^{\text {st }}$ line therapy, as augmenters, and in patients with anxiety due to psychoses and bipolar disorder. In this educational update, the results regarding symptoms, adverse effects, and functioning of these recent studies will be discussed. 